\title{
AN ANALYSIS TO THE TRAVELLING WAVE SOLUTION OF A DIFIUSIVE MODEL OF AN EPIDEMIC
}

\author{
M. S. ABUAL-RUB
}

\begin{abstract}
An SIR model of an epidemic with spatial spread is considered, an equilibrium and stability analysis has been done and then the behavior to the travelling wave solution of the mode! is obtained.
\end{abstract}

\section{Introduction}

The epidemic models have been initiated by Kermack and McKendrick[5]. Such models are useful in giving good estimates for the level of vaccination for the control of directly transmitted infectious diseases.

These epidemic models are often called SIR models because they describe a population which is partitioned into several distinct classes by an infection. In particular, the classes are the susceptibles (denoted by $S$ ), infectives (denoted by $I$ ), and individuals removed from the process (denoted by $R$ ). There are other classes of the population which are not considered in the SIR Models.

The Susceptibles is the class of the population who can catch the disease, the Infectives is the class of the population who have the disease and can transmit it, and the removed class have either had the disease, or are recovered, immune or isolated until recovered.

As mentioned before, the basic SIR (1927) model, which is given by Kermack and Mckendrick [5], assumed that (i) The gain of infective class is at a rate proportional to the number of infectives and susceptibles, that is $\alpha S I$, where $\alpha>0$ is a constant, and of course the susceptibles are lost at the same rate; (ii) the rate of removal of infectives to the removed class is proportional to the number of infectives, that is $\gamma I$ where $\gamma>0$ is a constant; (iii) The incubation period for which the susceptibles who contracts the disease became infectives is short enough to be negligible. Also, it is assumed that every pair of individuals has equal probability of coming into contact with one another.

Using all the above assumptions, the SIR model can be written as:

$$
\frac{d S}{d t}=-\alpha S I
$$

Received May 6, 1997.

1991 Mathematics Subject Classification. Primary 92Bxx, Secondary 92D30.

Key words and phrases. Epidemic, SIR model, spahal spread. 


$$
\begin{aligned}
& \frac{d I}{d t}=\alpha S I-\gamma I \\
& \frac{d R}{d t}=\gamma I
\end{aligned}
$$

where $\alpha>0$ is the infection rate and $\gamma>0$ is the removal rate of infectives. It is very clear, by adding equations (1.1)-(1.3), that:

$$
\frac{d S}{d t}+\frac{d I}{d t}+\frac{d R}{d t}=0
$$

This means that $S(t)+I(t)+R(t)=N$, where $N$ is the total size of the population.

An analysis to cquations (1.1)-(1.3) is considered in Murray [6], pp.612-616. Abualrub [1] has considered a model for which $S=S(x, t)$ and $I=I(x, t)$ with diffusion and an incubation period of $\sigma$ days to get:

$$
\begin{aligned}
& \frac{\partial S}{\partial t}-\Delta S=A-c I S \\
& \frac{\partial I}{\partial t}-\Delta I=c[I S(t)-I S(t-\sigma)]
\end{aligned}
$$

where $A, c$, and $\sigma$ are diffusion parameters.

Also in [1] p.26, $c$ is considered to be a function of $I$ and the local behavior of epidemics has becn analyzed in $L^{p, q}$ spaces. Now the $S I R$. model which we will consider here is laking into consideration spatial spread and this model has been considered by F. Hoppensteadt [4] p.68.

Fisher [3] and Baily [2] have considered a variety of problems and models for the spread and control of infectious diseases.

The model can be written as:

$$
\begin{aligned}
& \frac{\partial S}{\partial t}=-S\left[\alpha I+\beta \frac{\partial^{2} I}{\partial x^{2}}\right] \\
& \frac{\partial I}{\partial t}=S\left[\alpha I+\beta \frac{\partial^{2} I}{\partial x^{2}}\right]-\gamma I \\
& \frac{\partial R}{\partial t}=\gamma I
\end{aligned}
$$

where $-\infty<x<\infty, S=S(x, t), I=I(x, t)$ and $R=R(x, t)$.

\section{Equilibrium and Stability Analysis:}

Looking at equations (1.6)-(1.8), we have two possibilities for the steady states, namely (i) $\hat{S}=\hat{I}=0$ (ii) $\hat{S}=\frac{\gamma}{\alpha}, \hat{I}=0$.

Lets investigate both cases of steady states:

(i) $\hat{S}=\hat{I}=0$.

It is obvious that without diffusion, this state is unstable, i.e., if it is perturbed the infective and susceptible populations will deviate from the zero state. 
(ii) $\hat{S}=\frac{\gamma}{\alpha}, \hat{I}=0$.

This means that the infectives die out and all the population becomes susceptibles and removables which is possible.

Let $S=\frac{\gamma}{\alpha}+s, I=i$; where $s$ and $i$ are very small. Substitute into equations (1.6)-(1.8) and ignore non-linear terms to obtain:

$$
\begin{aligned}
& \frac{\partial s}{\partial t}=-\gamma i-\frac{\gamma \beta}{\alpha} \frac{\partial^{2} i}{\partial x^{2}} \\
& \frac{\partial i}{\partial t}=\frac{\gamma \beta}{\alpha} \frac{\partial^{2} i}{\partial x^{2}} \\
& \frac{\partial R}{\partial t}=\gamma i
\end{aligned}
$$

Lets consider equation (2.2) with $-\infty<x<\infty$ and suppose that $i(x, 0)=f(x)$; where $f(x)$ is bounded for all values of $x$ and integrable in every finite interval. The solution of the heat equation (2.2) will be:

$$
i(x, t)=\frac{\sqrt{\alpha}}{2 \sqrt{\gamma \beta \pi t}} \int_{-\infty}^{\infty} f(v) \exp \left\{\frac{-\alpha(x-v)^{2}}{4 \gamma \beta t}\right\} d v
$$

From (2.4) we may conclude that this state is locally stable.

\section{Travelling Wave Solutions:}

We now seek a travelling wave solution to the system (1.6)-(1.8) by setting $z=x-c t$; where $c$ is the wave velocity. We obtain the following equations:

$$
\begin{aligned}
c S^{\prime} & =S\left(\alpha I+\beta I^{\prime \prime}\right), \\
c I^{\prime} & =\gamma I-S\left(\alpha I+\beta I^{\prime \prime}\right), \\
c R^{\prime} & =-\gamma I
\end{aligned}
$$

where ${ }^{\prime}=d / d z$.

We will impose the following conditions:

$$
0 \leq S(-\infty)<S(\infty)=S_{0} ; \text { where } S_{0} \text { is a constant, } I(-\infty)=I(\infty)=0
$$

Lets now linearize equation (3.2) as $z \rightarrow \infty$ near the leading edge of the wave where $S \rightarrow S_{0}$ and $I \rightarrow 0$ to get:

$$
c I^{\prime} \approx \gamma I-S_{0}\left(\alpha I+\beta I^{\prime \prime}\right)
$$

we obtain solutions to (3.5) of the form:

$$
I(z) \sim O\left(\exp \left[\frac{-c \pm\left\{c^{2}+4 \gamma \beta S_{0}-4 \alpha \beta S_{0}^{2}\right\}^{1 / 2}}{2 \beta S_{0}}\right] z\right)
$$


In order for a travelling wave solution to exist and since we require $I(z) \rightarrow 0$ then using (3.6) we must have:

$$
c \geq 2\left(\alpha \beta S_{0}^{2}-\gamma \beta S_{0}\right)^{1 / 2} ; S_{0}>\frac{\gamma}{\alpha}
$$

Therefore from (3.7) we can say that the computed wave solution from the full non-linear system will involve into a travelling wave form with the minimum wave speed, namely,

$$
C_{\min }=2\left(\alpha \beta S_{0}^{2}-\gamma \beta S_{0}\right)^{1 / 2}
$$

Lets now linearize equation (3.1) as $z \rightarrow \infty$ by setting $S=S_{0}-s$; where $s$ is very small to get

$$
s^{\prime}=-\frac{S_{0}}{c}\left(\alpha I+\beta I^{\prime \prime}\right)
$$

Since $S=S_{0}-s$, the solution to (3.9) takes the form:

$$
S(z) \sim S_{0}+O\left(\exp \left[\frac{-\mathrm{c} \pm\left\{\mathrm{c}^{2}+4 \gamma \beta \mathrm{S}_{0}-4 \alpha \beta \mathrm{S}_{0}^{2}\right\}^{1 / 2}}{2 \beta \mathrm{S}_{0}}\right] z\right)
$$

Of course the same thing can be done to get the solution to (3.3) using (3.6).

\section{Conclusion}

From (3.7) we got $S_{0}>\frac{\gamma}{\alpha}$ or $\alpha S_{0}>\gamma$, this result is the most important condition here because if this condition is not satisfied there will be no progressing wave possible and this condition coincides with the result in F. Hoppensteadt [4], p.70. This result is interpreted as a lower bound on the population density of susceptibles for them to support a progressing epidemic.

\section{References}

[1] Abual-rub, M. S., Non-linear partial differential equations applied to diffusion problems arising in mathematical biology, Ph.D. Thesis, University of Illinois at Chicago, USA, 2530, 1992.

[2] Bailey, N. T. J., The mathematical theory of infectious discases, 2nd Ed., London; Griffin, 1975.

[3] Fisher, R. A., "The wave of advance of advantageous genes," Ann. Eugenics, 7(1937), 353-369.

[4] Hoppensteadt, F. C., Mathematical theories of popluations:demographics, genctics and epidemics, CBMS Lectures Vol. 20. Philadelphia: SIAM Publications, 1975.

[5] Kermack, W. O., McKendrick, A. G., "Contributions to the mathematical theory of epidemics," Proc. Roy. Soc. A 115(1927), 700-721; 138(1932), 55-83; 141(1933), 94-122.

[6] Murray, J. D., Mathematical Biology, Vol. 19, Biomathematics Texts, New York, Springer Verlag, 1989. 